\title{
Midterm Outcomes of Penetrating Keratoplasty Following Allogeneic Cultivated Limbal Epithelial Transplantation in Patients with Bilateral Limbal Stem Cell Deficiency
}

Junfa Xue

University of Jinan

\section{Dongfang Li}

Shandong Eye Institute \& Hospital

Yani Wang

Qingdao University

Chen Chen

Shandong University

Rufei Yang

Shandong Eye Institute \& Hospital

Qingjun Zhou

Shandong Eye Institute \& Hospital

Ting Liu

Shandong Eye Institute \& Hospital

Lixin Xie

Shandong Eye Institute \& Hospital

Yanling Dong ( $\nabla$ yanling1235@126.com )

Shandong Eye Institute \& Hospital

Research Article

Keywords: PK, CLET, LSCD, OSS, BCVA

Posted Date: January 8th, 2021

DOI: https://doi.org/10.21203/rs.3.rs-138752/v1

License: (c) (i) This work is licensed under a Creative Commons Attribution 4.0 International License. Read Full License 


\section{Abstract}

To evaluate the midterm outcomes of penetrating keratoplasty (PK) following allogeneic cultivated limbal epithelial transplantation (CLET) for bilateral total limbal stem cell deficiency (LSCD) \ten patients (10 eyes) with bilateral LSCD were enrolled in this prospective noncomparative case series study. Each participant received a PK approximately 6 months after a CLET. Topical tacrolimus, topical and systemic steroids, and oral ciclosporin were given postoperatively. Best-corrected visual acuity (BCVA), intraocular pressure (IOP), ocular surface grading scores (OSS), corneal graft epithelial rehabilitation, persistent epithelial defect (PED), immunological rejection, and graft survival rate were assessed. The time interval between PK and allogeneic CLET was $6.90 \pm 1.29$ (6-10) months. BCVA developed from 2.46 \pm 0.32 LogMAR preoperatively to 0.77 \pm 0.55 LogMAR post-PK $(P<0.001)$. Kaplan-Meier mean graft survival was $100 \%$ at 12,24 months, $80.0 \%$ at 36 months. PEDs appeared in 5 eyes at different periods post-PK, while graft rejection post-PK occurred in 4 eyes. Total OSS dropped from $12.4 \pm 4.4$ before allogeneic CLET to $1.4 \pm 1.5$ after PK. The sequential therapy of PK following allogeneic CLET can maintain a stable ocular surface with improved BCVA despite a relatively high graft rejection rate. Topical tacrolimus is effective in promoting PK survival post-CLET compared with previous reports.

\section{Introduction}

Corneal epithelial stem cells (SCs) are located at the palisades of Vogt and play a pivotal role in governing corneal epithelium renewal and regeneration ${ }^{1}$. Clinically, injuries to SCs can cause limbal stem cell deficiency (LSCD), manifesting as neovascularization, conjunctival transdifferentiation-induced conjunctivalization, corneal opacification, and persistent epithelial defect (PED) or recurrent erosions ${ }^{2,3}$. Among all the etiological factors, chemical injuries frequently cause severe total LSCD, termed when SCs are affected in all quadrants ${ }^{4-6}$, and cause many complications, such as secondary glaucoma, corneal scar, eyelid adhesion, etc.

Limbal stem cell transplantation has been useful for treating LSCD and reconstructing the ocular surface reported in recent decades $^{7,8,9}$. Given the effects of transplanting limbal tissue, cultivated SC transplantation has been vigorously advocated ${ }^{10,11}$. Burn injury patients often experience bilateral involvement, so autologous limbal SCs are not always available. Allogeneic culture of corneal limbal SCs ${ }^{12}$ as well as autologous culture of oral mucosal epithelium ${ }^{13,14}$ have become the primary cell source for cultured cell sheet transplantation. Previous reports demonstrated greater angiogenic properties of the oral epithelium than limbal cells $^{15,16}$, which may increase the graft failure ratio of keratoplasty operated on in the next step ${ }^{17}$. Thus, we selected allogeneic cultivated limbal epithelial transplantation (CLET) as the surgical method in phase one of this study ${ }^{18}$.

Clinical observations revealed that further penetrating keratoplasty (PK) surgery was required because of poor vision caused by stromal opacification, full-thickness scarring or endothelium deficiency ${ }^{19,20}$. Rather than combined surgery, a separate two-stage procedure several months after SC transplantation proved more recommended ${ }^{21}$. Our previous study reported that no allogeneic graft rejection occurred six months after $\mathrm{CLET}^{22}$, so we decided to perform a PK six months after allogeneic CLET to avoid multiple immunological irritations. To inhibit the allogenic rejection reaction, topical tacrolimus instead of ciclosporin was used after both CLET and PK ${ }^{23,24}$.

To date, little has been reported about the outcomes of PK following allogeneic CLET. Additionally, no information is available about the influence of topical tacrolimus on the survival of corneal grafts transplanted after allogeneic CLET. Therefore, we predesigned a sequential therapy for patients with total bilateral LSCD caused by chemical injuries in this study: an allogeneic CLET, a PK approximately six months later, with topical tacrolimus management during the course.

\section{Results}

The operative rate of PK in allogeneic CLET patients with total bilateral LSCD was 16.95\% (10/59 eyes, 10/50 patients). Eight male and two female patients conforming to the inclusion criterion undertook CLETs successfully, and then took further PKs. The full characteristics and clinical outcomes of the subjects are shown in Table 1. The mean patient age was $43.6 \pm 13.28$ years (2564 years). The mean follow-up period was $28.20 \pm 13.24$ months (12-54 months). All 10 eyes suffered from grade 3 burns according to the Roper Hall classifications. PKs were performed at mean $6.90 \pm 1.29$ months (6-10 months) after CLETs.

Page $2 / 15$ 
Table 1

Characteristics and clinical outcomes of enrolled patients.

\begin{tabular}{|c|c|c|c|c|c|c|c|c|c|c|}
\hline $\begin{array}{l}\text { Case } \\
\text { No. }\end{array}$ & $\begin{array}{l}\text { Age } \\
\text { range/ } \\
\text { Sex }\end{array}$ & Eye & Cause & $\begin{array}{l}\text { Previous } \\
\text { Surgery(ies) }\end{array}$ & $\begin{array}{l}F / U \\
(M)\end{array}$ & $\begin{array}{l}\text { Pre-CLET } \\
\text { BCVA } \\
\text { (LogMAR) }\end{array}$ & Interval(M) & $\begin{array}{l}\text { Post- } \\
\text { CLET } \\
\text { BCVA } \\
\text { (LogMAR) }\end{array}$ & $\begin{array}{l}\text { Post-PK } \\
\text { BCVA } \\
\text { (LogMAR) }\end{array}$ & Complication \\
\hline 1 & $20 \mathrm{~s} / \mathrm{M}$ & $\mathrm{OD}$ & Acid & $\begin{array}{l}\text { AMT } \\
\text { Lid surgery }\end{array}$ & 54 & 2.6 & 6 & 1.1 & 0.4 & Rej. \\
\hline 2 & $20 \mathrm{~s} / \mathrm{M}$ & os & Alkali & AMT & 42 & 2.2 & 7 & 1.2 & 0.5 & Rej. \\
\hline 3 & $30 \mathrm{~s} / \mathrm{M}$ & OS & Alkali & $\mathrm{N}$ & 22 & 2.7 & 6 & 2.2 & 0.6 & PED, Rej. \\
\hline 4 & $50 \mathrm{~s} / \mathrm{F}$ & OS & Acid & $\mathrm{N}$ & 38 & 2.3 & 10 & 2.7 & 0.4 & PED \\
\hline 5 & $40 \mathrm{~s} / \mathrm{M}$ & OS & Acid & AMT & 30 & 2.7 & 7 & 1.7 & 0.7 & PED \\
\hline 6 & $30 \mathrm{~s} / \mathrm{M}$ & OD & Acid & $\mathrm{N}$ & 29 & 2.7 & 6 & 2.6 & 2.2 & $\begin{array}{l}\text { Rej. } \\
\text { Twice,failure }\end{array}$ \\
\hline 7 & $60 \mathrm{~s} / \mathrm{M}$ & $\mathrm{OD}$ & Alkali & $\mathrm{N}$ & 20 & 1.7 & 7 & 2.1 & 1.0 & $\begin{array}{l}\text { PED, } \\
\text { infection }\end{array}$ \\
\hline 8 & $30 \mathrm{~s} / \mathrm{F}$ & OS & Alkali & $\begin{array}{l}\text { AMT } \\
\text { Lid surgery }\end{array}$ & 14 & 2.7 & 8 & 2.4 & 0.5 & $\mathrm{~N}$ \\
\hline 9 & $50 \mathrm{~s} / \mathrm{M}$ & os & Acid & AMT & 21 & 2.4 & 6 & 1.1 & 0.4 & PED \\
\hline 10 & $60 \mathrm{~s} / \mathrm{M}$ & OS & Alkali & Lid surgery & 12 & 2.6 & 6 & 1.2 & 1.0 & $\mathrm{~N}$ \\
\hline
\end{tabular}

The OSS outcomes are summarized in Fig. 2 (A-G). Among the parameters, only corneal neovascularization (Fig. 2A) and opacification grading scores (Fig. $2 \mathrm{C}$ ) were not improved after allogeneic CLET ( $>0.05$ ) but were improved significantly after PK $(P<0.001)$.

The mean BCVA was $2.46 \pm 0.32$ LogMAR at baseline (equal to finger count $20 \mathrm{~cm}$ ) (Fig. 2H). The mean BCVA post CLET (measured when rehospitalized for PK) was $1.83 \pm 0.65$ LogMAR (equal to 20/160). The mean BCVA post-PK increased to $0.77 \pm$ 0.55 LogMAR (equal to 20/100) $\left(X^{2}=21.633, P<0.001\right)$. The mean BCVA change throughout the whole treatment course was $1.68 \pm 0.63$ LogMAR (Cl: $-2.00 \sim-1.27$ ) (Fig. 2I), which is consistent with the results of the neovascularization, conjunctivalization and opacification grading scores. All the enrolled eyes had noticeable vision improvement.

All the enrolled eyes had a certain range of corneal graft epithelial defects after PK surgery, wholly healed in 2-10 days (mean 6.6 \pm 2.4 days) with topical medication bandage lens application. PEDs occurred in 5/10 cases (Table 1, Fig. 3 ). The PED-free graft survival rates at 12,24 , and 36 months were $70,43.8$, and $43.8 \%$, respectively.

Four (40\%) patients had endothelial graft rejection(s) at the second stage after PK (Fig. 3). The rejection-free graft survival rates at 12 and 24 months were 90.0 and $75.0 \%$, respectively. One patient (case 6) with a history of diabetes had two episodes of graft rejection after PK. The other three patients had no discernible causative factors. When rejection occurred, those eyes had redness, irritation, and photophobia, and visual acuity declined thereafter.

Nine out of ten grafts survived (BCVA increased, opacification grade 0 or 1 at the central $6 \mathrm{~mm}$ of corneal graft) by the time of the last visit (Fig. 3, Supplemental Fig. 1). Kaplan-Meier mean graft survival was $100 \%$ at 12, 24 months, $80.0 \%$ at 36 months. One diabetic patient (case 6) had two episodes of rejection. He discontinued the anti-immune therapy by self-determination and was found to relapse at 29 months past PK. Slight peripheral vascularization (grade 1) occurred in some other eyes initially from 2-6 months after PK. Mild opacity accompanied by a few vessels at the edge of the graft was noted in some subjects. Two eyes 
(cases 6 and 9) underwent ECCE simultaneously with PK. Case 6 was implanted with an intraocular lens, while case 9 was aphakic thereafter. Two cases (cases 3 and 9 ) had high postoperative IOP controlled by medication ( $2 \%$ atenolol). Case 7 was diagnosed with Citrobacter flautii conjunctival sac infection 3 months after PK, followed by a recurrent corneal graft ulcer. He received topical medication as well as blepharoplasty and eventually healed with a transparent cornea graft. Figure 4 shows the changes in the ocular surface of Case 2 during the entire treatment (details in the figure legend).

ALL the corneal buttons sectioned after PK were subjected to hematoxylin and eosin (HE), periodic acid-Schiff (PAS), and immunohistochemistry staining. HE staining demonstrated normal polarity with cell layers similar to the normal cornea but with more layers of epithelial cells ${ }^{25}$, and new vessels appeared in the deep stroma (Fig. 5A). PAS staining showed no obvious goblet cells or conjunctivalization present on the corneal button (Fig. 5B). Positive staining with K3 was shown in the suprabasal and superficial corneal epithelium (Fig. 5C). Sporadic staining with P63 was detected in the basal and superabasal layer of epithelium of 6 buttons (Fig. 5D). This pattern was similar to that of the normal limbal cornea epithelium (Fig. 5E, F, G, H)

\section{Discussion}

Among all the causes of LSCD, chemical injuries frequently occur in both eyes. Thus, autologous limbal SCs from the healthy fellow eye cannot be transplanted. Other cell source options, such as oral mucosal SCs and allogeneic SCs, can be selected. We chose allogeneic CLET because of its superiority in maintaining epithelial graft integrity ${ }^{18}$ and avascular properties ${ }^{15,16}$. We used limbal cells from the corpse, as Shimazaki et al. ${ }^{26}$ reported no significant differences in stem cell transplantation between using limbal tissue from cadavers and living relatives. Limbal SC transplantation is performed near the conjunctival area, adjacent to blood vessels and lymph. Therefore, immunity-free advantages inherent to central keratoplasty are lost, increasing the likelihood of immune rejection ${ }^{27}$, especially when allogeneic cell sheets are utilized for severe total LSCD patients. To improve visual acuity and remove full-thickness scarring, we performed PK surgeries approximately 6 months later, although providing another allograft antigen simultaneously. This approach naturally raises questions: under multiple allogeneic tissue or cell stimulation, will the risk of graft rejection or failure increase? If yes, is this rejection controllable? Based on these questions, we carried out our research.

Allogeneic CLET provided a stable and favorable condition for further PK surgery. Shimazaki et al. ${ }^{28}$ defined successful ocular surface reconstruction as increased corneal transparency, reduced or absent corneal vascularization, a smooth corneal surface with a stable epithelium, and a corneal phenotype on the central cornea with and without peripheral conjunctival invasion, which is consistent with our results. In the current study, all parameters of the enrolled eyes were optimized after allogeneic CLET except (stromal) neovascularization and opacification, according to the OSSs (Fig. 2). Because many vessels invading into the stroma cannot be corrected by dissection of the superficial fibrovascular pannus in allogeneic CLET, which was part of the reason why we addressed them in an additional PK procedure, neovascularization before keratoplasty may increase graft failure and rejection risk $^{17}$. PK removed the central corneal tissues, including the invasive vessels, and improved visual acuity by approximately 2 lines postoperatively $(P<0.001)$. Reports showed that the final success rate of ocular surface reconstruction was similar by using autologous limbal cells, cadaveric limbal cells, and living relatives' limbal cells ${ }^{26,29}$. We used discarded donor corneoscleral rings after corneal grafting (Fig. 1) ${ }^{22}$, which dramatically avoids additional pain and invasiveness to living donors and improved the utilization efficiency of donated corneas. As ocular reconstruction simultaneously combined with PK had a high rate of failure, we separated the project into two steps and assigned PK approximately 6 months later ${ }^{30}$.

The irreversible immune rejection of corneal grafts is the primary cause of allograft failure in the intermediate and late periods after surgery ${ }^{31}$. The rejection rates at 12,24 , and 36 months were $10 \%, 25 \%$, and $61 \%$ in the current study. This was higher than the results of high-risk penetrating keratoplasty in 5 years reported by Chow SP ${ }^{32}$ but was lower than that in 2 years reported by Szaflik $\mathrm{JP}^{33}$. A failure rate due to graft rejection (25\%) was lower than them in the current study. The pioneering case of rejection occurred 7 months after PK, and other rejections appeared at various stages after PK (Fig. 4). The failure case encountered two episodes of

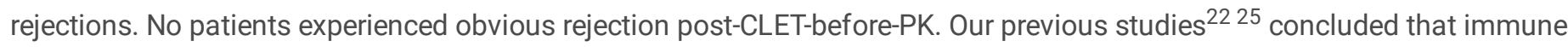
rejection after allogeneic CLET mainly occurred in the first six months. DNA analysis from our institute ${ }^{25}$ and others ${ }^{34} 35$ reported that as early as three months after allogeneic CLET, no donor information was found on the recipient ocular surface. Additionally, the corneal buttons sectioned after PK showed epithelial integrity similar to the normal cornea surface with no recurrent conjunctivalization in this series (Fig. 5). The ocular surface progressed to a relatively stable condition based on the OSS results 
(Fig. 2). Thus, allogeneic CLET can provide long-term benefits that do not necessarily depend on donor cell survival. The exact mechanism of the high rejection rate is still unclear. Immune rejection after PK under this circumstance should be attributed more to variable ocular status, superficial ocular inflammation in different individuals, and chronic recipient-to-donor corneal graft immune reaction rather than doubled allogeneic cells or graft stimulation.

Based on this, various cell sources for sheet transplantation should theoretically contribute to almost the same graft rejection levels after PK if the interval after ocular surface reconstruction is sufficiently long. Alireza Baradaran-Rafii ${ }^{13}$ reported that graft rejection occurred in 33.3\% of PK patients after COMET, while one patient (all fourteen) failed due to graft vascularization caused by PED and bacterial keratitis. Figueiredo GS et al. ${ }^{36}$ described that graft rejection occurred in $30 \%$ of cases of PK following autologous CLET, with $50 \%$ leading to graft failure. All of these reported failure cases were somehow related to graft rejection, indicating the need for an effective anti-rejection scheme.

It is noteworthy that $9 / 10$ cases of this sequential therapy maintained a clear center (opacification grade 0 or $1^{18,37}$ ) by the last visit, which was considered the success/survival criterion for the strategy. However, previous studies reported low survival rates of similar projects ${ }^{20,34}$ (Table 2). The difference lies in the development of anti-rejection drugs, especially the widespread application of tacrolimus in the clinic ${ }^{24,38-40}$, which led to the controllability of transplant rejection strengthened. By the continuous use of topical tacrolimus and low-concentration hormones on the ocular surface, the immune response after PK can be controlled in a timely and effective manner. In particular, patients were strongly recommended rehospitalization to receive an anti-rejection booster therapy (including systemic hormonotherapy and anti-immune therapy) as soon as post-PK rejection was found ${ }^{18}$. All of these manners were intended to control the rejection to an extreme. Compared with earlier related reports, the utility of topical tacrolimus seems more practical and recommendable than previous drug treatments (topical ciclosporin, for example) according to our midterm results (Fig. 3, Table 2).

Table 2

A comparison of the methods and outcomes of the current study with previously reports of PK after allogeneic CLET for bilateral LSCD

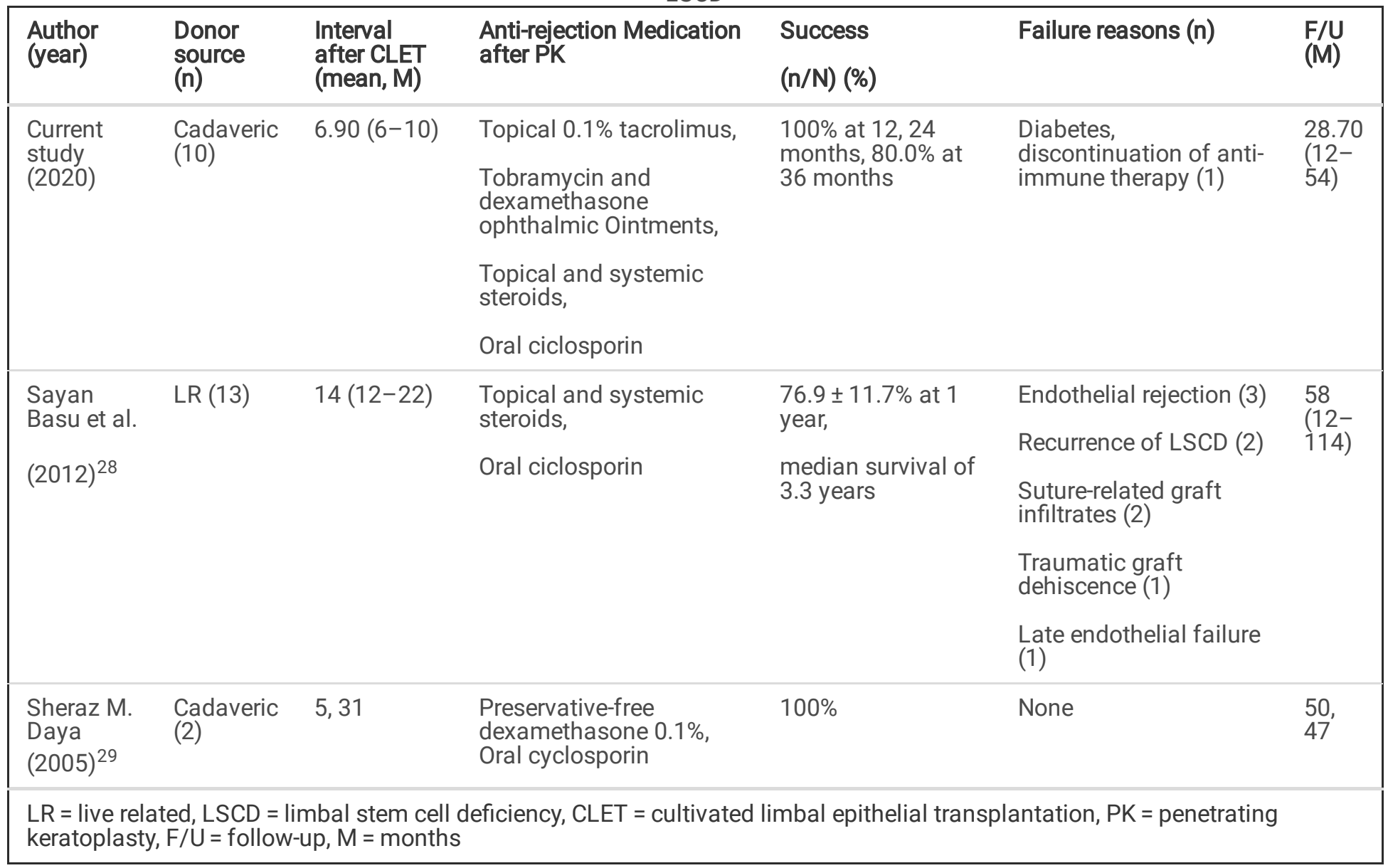


We also observed mild vascularization of the corneal limbus 2-6 months after PK, which is similar to the COMET + PK scenario ${ }^{13}$. More apparent peripheral vascularization was noted in the patient with repeated rejection (case 6). Reports indicate that both graft rejection and neovascularization can adversely affect the graft survival rate ${ }^{41}$. BCVA declined at least one line after rejection. However, compared to preoperative BCVA, post-PK BCVA was improved at the last visit. Thus, BCVA was not severely compromised in patients with mild peripheral vascularization. The possible reason may be that the ocular surface was in a quiescent noninflammatory state before PK in these chemical injury patients.

PED is another severe trouble to address after PKs. If the graft cannot maintain complete epithelization after transplantation, it will dissolve, perforate, develop edema or infection, and eventually fail. Five eyes (50\%) grew PED at various periods in our study (Table 1, Fig. 3) and healed fortunately without noticeable corneal scarring or opacification. Post-PK epithelial problems were also observed in the COMET + PK project ${ }^{13}$ but were not mentioned in the autologous CLET + PK research ${ }^{36}$. The morbidity of PED should be attributed to the barely satisfactory ocular surface conditions of burn-injured patients, such as hypophasis, insufficient tear secretion, or inadequate tear film quality.

Our outcomes must be considered under circumstances of these limitations: small numbers of samples studied, limited follow-up period, and test error in manual evaluation. The limited sample size did not allow for the establishment of subgroups; thus, $P$ values should be interpreted with caution. Moreover, variability in inclusion criteria, culture techniques, surgical procedures, outcome measurements, and examination errors of the reported results during the follow-up should be noted. The limitations above are integrally encountered by all teams working in the field.

In conclusion, this study demonstrates the encouraging effects of the sequential therapy of PK following a previous allogeneic CLET in promoting visual rehabilitation and maintaining ocular stability for patients with total bilateral LSCD caused by chemical burns. The allogeneic CLET offered a useful preparation for further PK. However, it also reminds us that under the stimulation of allogeneic stem cells and allograft, graft rejection post-PK. In contrast to previous similar studies, the application of tacrolimus and other related immunosuppressants increased graft survival. We believe these have huge implications, and further research should help uncover them.

\section{Methods}

\section{Subjects}

A single-center prospective noncomparative case series study was conducted. This study was approved by the ethics committee of Shandong Eye Institute and adhered to the Declaration of Helsinki's tenets for human subject study. The clinical trial was registered on the Chinese Clinical Trial Registry (www.chictr.org.cn), and the registration number is ChiCTR2000032536 (Retrospective registration date: 1/5/2020). A cohort of adults with chronic ocular complications from chemical injuries was recruited at Qingdao Eye Hospital between June 15, 2016, and June 16, 2020. An informed consent was obtained from all participants.

Inclusion criteria were as follows: (1) bilateral total LSCD secondary to chemical burns, (2) age $\geq 18$ years, (3) $\geq 6$ months after injury, with regular eyelids, ocular surface in homeostasis, (4) full-layer corneal stromal opacification, scars, or swelling after allogeneic CLET with BCVA less than 20/200, and (5) follow-up of $\geq 12$ months after PK. The exclusion criteria were as follows: (1) previous limbal tissue transplantation or other intraocular surgeries and (2) severe systemic disease(s) or contraindications for steroids and immunosuppressants. Fifty patients (59 eyes) with severe LSCD underwent allogeneic CLET and were followed up for at least 12 months, 10 of whom (10 eyes) underwent subsequent PK approximately six months later (graphical procedure of the whole sequential therapy in Fig. 1).

Patients underwent full ophthalmic examination, including best-corrected visual acuity (BCVA), intraocular pressure (IOP), optical coherence tomography (OCT), slit-lamp assessment of ocular surface grading scores (OSS, according to Chie Sotozono's ocular surface grading criteria ${ }^{18,37}$ ), corneal graft epithelial rehabilitation, persistent epithelial defect (PED), immunological rejection, and graft survival rate during the process. Successful CLET before PK was defined by reduced or absent corneal vascularization, a smooth corneal surface with a stable epithelium and a corneal phenotype on the central cornea with and without peripheral 
conjunctival invasion. Successful PK following allogeneic CLET was clinically defined by an intact epithelized, stable corneal surface, with increased corneal transparency (opacification grade 0 or 1 )and improved BCVA. Corneal graft failure was defined as central corneal (center diameter 6 millimeters) revascularization, reconjunctivalization or a loss of central graft clarity (opacification grade 2 or more).

\section{Allogeneic limbal epithelial culture and CLET}

Consistent with our previous research ${ }^{18,22}$, limbal SCs were acquired from the residual cadaveric donor corneoscleral ring after keratoplasty. Donors' infectious disease test results were negative. Specimens were cut into granules, inoculated on an amniotic membrane (AM) spreading on the bottom of transwell culture inserts, and then co-cultured with mitomycin $\mathrm{C}(\mathrm{MMC})$-inactivated $\mathrm{NIH} 3 \mathrm{~T} 3$ fibroblasts for at least 5 days until confluence, with the medium changed every 2 days. Dulbecco's modified Eagle's medium/F-12 (3:1) supplemented with 10\% fetal bovine serum (Gibco, Grand Island, NY), 2 nM 3,3'5-triiodo-L-thyronine sodium salt (Sigma), 1\% nonessential amino acids (Invitrogen, Carlsbad, CA), $0.1 \mathrm{nM}$ cholera toxin (Sigma, St. Louis, MO), insulintransferrin selenium (Invitrogen, Carlsbad, CA), $0.4 \mathrm{ng} / \mathrm{mL}$ hydrocortisone succinate (Wako, Osaka, Japan), 2 mM L-glutamine (Invitrogen), penicillin-streptomycin (HyClone, Logan, UT) and $10 \mathrm{ng} / \mathrm{mL}$ recombinant human epithelial growth factor (R\&D Systems, Minneapolis, MN) were used for the culture of limbal epithelial cells.

The environment of the incubation was $37{ }^{\circ} \mathrm{C}$ with $5 \%$ carbon dioxide and $95 \%$ air. AMs with incubated cells stratifying into $3-5$ layers were finally assigned to clinical application.

All operating procedures were performed under peribulbar anesthesia by two experienced surgeons (LXX and YLD). All operating procedures were performed under peribulbar anesthesia by two experienced surgeons (XLX, YLD). Initially, 360-degree conjunctival peritomy, dissection of the symblepharon, recession of the bulbar conjunctiva, dissection of the fibrovascular pannus on the corneal surface and subconjunctival fibrotic tissues were performed carefully. Second, the graft of the cultivated cell sheet was placed onto the bare sclera and corneal stroma with the epithelial side upward and intermittently sutured with 10-0 nylon sutures. A conjunctival sac was rebuilt by suturing the residual conjunctiva tissue outside the limbus. Finally, a bandage contact lens (Bausch and Lomb, NY, USA) was applied on the ocular surface, and tobramycin and dexamethasone ophthalmic ointments were applied superficially.

\section{Penetrating keratoplasty}

Classical optical PK surgeries corresponding to our previously published research ${ }^{42}$ were performed approximately 6 months after allogeneic CLET for patients fulfilling the inclusion criteria of PK Superior fresh donor corneal grafts from an eye bank were used for transplantation to counterbalance the defect in the compromised ocular surface. The recipient cornea was trephined with a 7.25-7.75-mm trephine, and the donor tissue was fabricated with a diameter $0.25 \mathrm{~mm}$ larger than the diameter of the recipient graft site. If needed, $250 \mathrm{~mL}$ of $20 \%$ mannitol containing $10 \mathrm{mg}$ of dexamethasone was given intravenously once or twice after PK to control intraocular pressure (IOP) and suppress irritation from intraocular inflammation. Corneal grafts were secured to the recipient bed with 16 interrupted 10-0 nylon sutures. Similar to CLET, a bandage contact lens was placed on the ocular surface after PK. The recipient corneal buttons were sectioned and subjected to hematoxylin and eosin (HE), periodic acid-Schiff (PAS), and immunohistochemistry staining.

\section{Immunohistochemistry and antibodies}

All procedures were consistent with our previous research ${ }^{25}$. Primary antibodies included anti-CK3 antibody (epithelium marker, 1:200 dilution, Abcam, USA) and anti-P63 antibody (myoepithelial cell marker, 1:50 dilution, Proteintech, USA). The experiments reported in this study were reproducible.

\section{Postoperative management and follow-up schedule}

Post-PK medication was similar to that after allogeneic CLET ${ }^{18,22}$.Intravenous methylprednisolone (2 mg/kg) was injected daily for 3-5 days immediately after transplantation. Then, oral prednisolone $(1 \mathrm{mg} / \mathrm{kg})$ was administered daily and tapered over $2-3$ months to avoid allograft rejection. Autologous serum eyedrops containing $0.02 \mathrm{mg}$ dexamethasone and $0.1 \mathrm{mg}$ tobramycin per milliliter were used every $2 \mathrm{~h}$ for the first week. Eyedrops containing $0.1 \%$ fluorometholone, $0.5 \%$ levofloxacin eyedrops, and preservative-free artificial tears were applied four times daily for the next 2 weeks. Tobramycin and dexamethasone ophthalmic

Page $7 / 15$ 
ointments were applied topically every night. $0.1 \%$ tacrolimus was administered 4 times daily after corneal epithelial integration. The aforementioned topical treatment should be accommodated depending on clinical status after 3 weeks. Therapeutic bandage contact lenses were used to preserve the graft epithelium for 1-3 months after surgery. Patients were inspected daily during the first week after surgery, weekly during the first month, and then monthly.

All patients underwent thorough examinations of both eyes at every follow-up. When a PED was found during the follow-up, autologous serum eyedrops and bandage contact lenses were applied for ocular surface healing and protection. Moreover, if this strategy was unproductive for more than one month, AM transplantation or even tarsorrhaphy was prefered. Patients were promptly rehospitalized to take an anti-immune booster therapy on the occasion that rejective symptoms appeared and treated with approaches similar to those immediately after PK surgery (topical tacrolimus and fluorometholone, systemic hydrocortisone, prednisone, or cyclosporine if necessary). Any patients with PEDs and simultaneous severe conjunctival congestion were also treated with an anti-rejection therapy schedule. Topical $0.1 \%$ tacrolimus (Senju, Japan) was administered from allogeneic CLET to PK and continued for at least 2 years after PK. Topical $0.02 \%$ fluorometholone was maintained along with immunosuppressants.

\section{Data collection}

Data were documented in a predesigned format at every follow-up and recorded as a completed form. The data included the patient case number, age, sex, the affected eye, the type and date of injury, details of previous ocular procedures, follow-up duration, Snellen BCVA (before CLET, after CLET, and after PK), IOP, the presence or absence of palpebral fissure abnormalities, tear secretion, OSSs, other surgical details, postoperative complications, and the ocular surface condition at each visit (detected by slitlamp examination and fluorescein staining).

\section{Statistical analysis}

BCVA was modified into LogMAR units for statistical analysis. According to a previous study ${ }^{13}$, LogMAR BCVA less than 20/200 was regarded as 2.6, 2.7, 2.8, and 2.9 for counting fingers, hand motion, light perception, and no light perception, respectively. All data were analyzed using SPSS statistical software (version 20.0; IBM Corp., Chicago, IL, USA). The mean \pm SD of quantitative variables was computed using Student's t test. Homoscedasticity of BCVA and OSSs during the strategy was computed. When the results were homogeneous, one-way ANOVAs were performed, whereas Kruskal-Wallis tests were performed if the results were not homogeneous. Kaplan-Meier survival analysis was performed to evaluate corneal PED-free survival, graft rejection-free survival and successful graft survival after PK. A P-value of less than 0.05 was determined to be statistically significant, and a value less than 0.01 was considered very significant.

\section{Declarations}

\section{Acknowledgements}

This study was supported by the Academic Promotion Program and Innovation Project of Shandong First Medical University (2009ZL001). The authors thank Junyi Wang, Zhen Guo, Hua Li, Xia Qi, Xiaoyun Wang and Qianqian Kong (Shandong Eye Institute) for help with the data collection, statistical analysis, and instrument use.

\section{Author contributions}

Junfa Xue: Analyzed data, did literature search, and wrote the manuscript. Dongfang Li, Rufei Yang, Ting Liu: Measured and collected the OSSs and as-OCT. Yani Wang, Chen Chen and Qingjun Zhou: joined the discussion of the research and helped analyzed data. Yanling Dong \& Lixin Xie: Designed this research, analyzed data, and revised the manuscript.

\section{Competing interests}

The authors declare no competing interests.

\section{References}


1. Schermer, A., Galvin, S. \& Sun, T. T. Differentiation-related expression of a major $64 \mathrm{~K}$ corneal keratin in vivo and in culture suggests limbal location of corneal epithelial stem cells. The Journal of cell biology. 103, 49-62 https://doi.org/10.1083/jcb.103.1.49 (1986).

2. Hou, L. et al. Agrin Promotes Limbal Stem Cell Proliferation and Corneal Wound Healing Through Hippo-Yap Signaling Pathway. Investigative ophthalmology \& visual science. 61, 7 https://doi.org/10.1167/iovs.61.5.7 (2020).

3. Le, Q., Yang, Y., Deng, S. X. \& Xu, J. Correlation between the existence of the palisades of Vogt and limbal epithelial thickness in limbal stem cell deficiency. Clinical \& experimental ophthalmology. 45, 224-231 https://doi.org/10.1111/ceo.12832 (2017).

4. Shanbhag, S. S. et al. Autologous limbal stem cell transplantation: a systematic review of clinical outcomes with different surgical techniques. The British journal of ophthalmology. 104, 247-253 https://doi.org/10.1136/bjophthalmol-2019-314081 (2020).

5. Vazirani, J. et al. Limbal Stem Cell Deficiency-Demography and Underlying Causes. American journal of ophthalmology. 188, 99-103 https://doi.org/10.1016/j.ajo.2018.01.020 (2018).

6. Deng, S. X. et al. Global Consensus on Definition, Classification, Diagnosis, and Staging of Limbal Stem Cell Deficiency. Cornea. 38, 364-375 https://doi.org/10.1097/IC0.0000000000001820 (2019).

7. Kenyon, K. R. \& Tseng, S. C. Limbal autograft transplantation for ocular surface disorders. Ophthalmology. 96, 709-722 discussion 722 - 703 https://doi.org/10.1016/s0161-6420(89)32833-8 (1989).

8. Pellegrini, G. et al. Long-term restoration of damaged corneal surfaces with autologous cultivated corneal epithelium. Lancet. 349, 990-993 https://doi.org/10.1016/S0140-6736(96)11188-0 (1997).

9. Shanbhag, S. S., Saeed, H. N., Paschalis, E. I. \& Chodosh, J. Keratolimbal allograft for limbal stem cell deficiency after severe corneal chemical injury: a systematic review. The British journal of ophthalmology. 102, 1114-1121 https://doi.org/10.1136/bjophthalmol-2017-311249 (2018).

10. Sacchetti, M., Rama, P., Bruscolini, A. \& Lambiase, A. Limbal Stem Cell Transplantation: Clinical Results, Limits, and Perspectives. Stem cells international 2018, 8086269, doi:10.1155/2018/8086269 (2018).

11. Borderie, V. M. et al. Long-Term Results of Cultured Limbal Stem Cell Versus Limbal Tissue Transplantation in Stage III Limbal Deficiency. Stem cells translational medicine. 8, 1230-1241 https://doi.org/10.1002/sctm.19-0021 (2019).

12. Zakaria, N. et al. Results of a phase I/II clinical trial: standardized, non-xenogenic, cultivated limbal stem cell transplantation. Journal of translational medicine. 12, 58 https://doi.org/10.1186/1479-5876-12-58 (2014).

13. Baradaran-Rafii, A. et al. Midterm outcomes of penetrating keratoplasty after cultivated oral mucosal epithelial transplantation in chemical burn. The ocular surface. 15, 789-794 https://doi.org/10.1016/j.jtos.2017.08.006 (2017).

14. Burillon, C. et al. Cultured autologous oral mucosal epithelial cell sheet (CAOMECS) transplantation for the treatment of corneal limbal epithelial stem cell deficiency. Investigative ophthalmology \& visual science. 53, 1325-1331 https://doi.org/10.1167/iovs.11-7744 (2012).

15. Kanayama, S. et al. Analysis of angiogenesis induced by cultured corneal and oral mucosal epithelial cell sheets in vitro. Experimental eye research. 85, 772-781 https://doi.org/10.1016/j.exer.2007.08.011 (2007).

16. Kanayama, S. et al. Analysis of soluble vascular endothelial growth factor receptor-1 secreted from cultured corneal and oral mucosal epithelial cell sheets in vitro. The British journal of ophthalmology. 93, 263-267 https://doi.org/10.1136/bjo.2008.141580 (2009).

17. Bachmann, B., Taylor, R. S. \& Cursiefen, C. Corneal neovascularization as a risk factor for graft failure and rejection after keratoplasty: an evidence-based meta-analysis. Ophthalmology 117, 1300-1305 e1307, doi:10.1016/j.ophtha.2010.01.039 (2010).

18. Wang, J. et al. Comparison of the efficacy of different cell sources for transplantation in total limbal stem cell deficiency. Graefe's archive for clinical and experimental ophthalmology = Albrecht von Graefes Archiv fur klinische und experimentelle Ophthalmologie. 257, 1253-1263 https://doi.org/10.1007/s00417-019-04316-z (2019).

19. Kolli, S., Ahmad, S., Lako, M. \& Figueiredo, F. Successful clinical implementation of corneal epithelial stem cell therapy for treatment of unilateral limbal stem cell deficiency. Stem cells. 28, 597-610 https://doi.org/10.1002/stem.276 (2010). 
20. Basu, S. et al. Clinical outcomes of xeno-free allogeneic cultivated limbal epithelial transplantation for bilateral limbal stem cell deficiency. The British journal of ophthalmology. 96, 1504-1509 https://doi.org/10.1136/bjophthalmol-2012-301869 (2012).

21. Basu, S. et al. Clinical outcomes of penetrating keratoplasty after autologous cultivated limbal epithelial transplantation for ocular surface burns. American journal of ophthalmology 152, 917-924 e911, doi:10.1016/j.ajo.2011.05.019 (2011).

22. Qi, X., Xie, L., Cheng, J., Zhai, H. \& Zhou, Q. Characteristics of immune rejection after allogeneic cultivated limbal epithelial transplantation. Ophthalmology. 120, 931-936 https://doi.org/10.1016/j.ophtha.2012.11.001 (2013).

23. Abudou, M., Wu, T., Evans, J. R. \& Chen, X. Immunosuppressants for the prophylaxis of corneal graft rejection after penetrating keratoplasty. The Cochrane database of systematic reviews. CD007603, https://doi.org/10.1002/14651858.CD007603.pub2 (2015).

24. Magalhaes, O. A., Marinho, D. R. \& Kwitko, S. Topical $0.03 \%$ tacrolimus preventing rejection in high-risk corneal transplantation: a cohort study. The British journal of ophthalmology. 97, 1395-1398 https://doi.org/10.1136/bjophthalmol-2013-303639 (2013).

25. Chen, P. et al. Characterization of the corneal surface in limbal stem cell deficiency and after transplantation of cultured allogeneic limbal epithelial cells. Graefe's archive for clinical and experimental ophthalmology = Albrecht von Graefes Archiv fur klinische und experimentelle Ophthalmologie. 254, 1765-1777 https://doi.org/10.1007/s00417-016-3410-2 (2016).

26. Shimazaki, J. et al. Transplantation of human limbal epithelium cultivated on amniotic membrane for the treatment of severe ocular surface disorders. Ophthalmology. 109, 1285-1290 https://doi.org/10.1016/s0161-6420(02)01089-8 (2002).

27. Satake, Y. et al. Immunological rejection following allogeneic cultivated limbal epithelial transplantation. JAMA ophthalmology. 131, 920-922 https://doi.org/10.1001/jamaophthalmol.2013.15 (2013).

28. Shimazaki, J. et al. Factors influencing outcomes in cultivated limbal epithelial transplantation for chronic cicatricial ocular surface disorders. American journal of ophthalmology. 143, 945-953 https://doi.org/10.1016/j.ajo.2007.03.005 (2007).

29. Shimazaki, J. et al. Long-term outcomes of cultivated cell sheet transplantation for treating total limbal stem cell deficiency. The ocular surface. https://doi.org/10.1016/j.jtos.2020.06.005 (2020).

30. Solomon, A. et al. Long-term outcome of keratolimbal allograft with or without penetrating keratoplasty for total limbal stem cell deficiency. Ophthalmology. 109, 1159-1166 https://doi.org/10.1016/s0161-6420(02)00960-0 (2002).

31. Panda, A., Vanathi, M., Kumar, A., Dash, Y. \& Priya, S. Corneal graft rejection. Survey of ophthalmology. 52, 375-396 https://doi.org/10.1016/j.survophthal.2007.04.008 (2007).

32. Chow, S. P., Cook, S. D. \& Tole, D. M. Long-Term Outcomes of High-Risk Keratoplasty in Patients Receiving Systemic Immunosuppression. Cornea. 34, 1395-1399 https://doi.org/10.1097/IC0.0000000000000615 (2015).

33. Szaflik, J. P., Major, J., Izdebska, J., Lao, M. \& Szaflik, J. Systemic immunosuppression with mycophenolate mofetil to prevent corneal graft rejection after high-risk penetrating keratoplasty: a 2-year follow-up study. Graefe's archive for clinical and experimental ophthalmology = Albrecht von Graefes Archiv fur klinische und experimentelle Ophthalmologie. 254, 307-314 https://doi.org/10.1007/s00417-015-3200-2 (2016).

34. Daya, S. M. et al. Outcomes and DNA analysis of ex vivo expanded stem cell allograft for ocular surface reconstruction. Ophthalmology. 112, 470-477 https://doi.org/10.1016/j.ophtha.2004.09.023 (2005).

35. Henderson, T. R., Coster, D. J. \& Williams, K. A. The long term outcome of limbal allografts: the search for surviving cells. The British journal of ophthalmology. 85, 604-609 https://doi.org/10.1136/bjo.85.5.604 (2001).

36. Figueiredo, G. S. et al. Outcomes of Penetrating Keratoplasty Following Autologous Cultivated Limbal Epithelial Stem Cell Transplantation. Stem cells. 36, 925-931 https://doi.org/10.1002/stem.2803 (2018).

37. Sotozono, C. et al. New grading system for the evaluation of chronic ocular manifestations in patients with Stevens-Johnson syndrome. Ophthalmology. 114, 1294-1302 https://doi.org/10.1016/j.ophtha.2006.10.029 (2007).

38. Zhai, L. Y., Zhang, X. R., Liu, H., Ma, Y. \& Xu, H. C. Observation of topical tacrolimus on high-risk penetrating keratoplasty patients: a randomized clinical trial study. Eye. 34, 1600-1607 https://doi.org/10.1038/s41433-019-0717-3 (2020).

39. Joseph, A., Raj, D., Shanmuganathan, V., Powell, R. J. \& Dua, H. S. Tacrolimus immunosuppression in high-risk corneal grafts. The British journal of ophthalmology. 91, 51-55 https://doi.org/10.1136/bjo.2006.097428 (2007).

Page 10/15 
40. Zamos, D. T. \& Tabin, G. Tacrolimus (FK506) for high-risk corneal and limbal grafts. Ophthalmology 109 1953; author reply 1953, doi:10.1016/s0161-6420(02)01214-9 (2002).

41. Guilbert, E. et al. Long-term rejection incidence and reversibility after penetrating and lamellar keratoplasty. American journal of ophthalmology 155, 560-569 e562, doi:10.1016/j.ajo.2012.09.027 (2013).

42. Xie, L., Dong, X. \& Shi, W. Treatment of fungal keratitis by penetrating keratoplasty. The British journal of ophthalmology. $\mathbf{8 5}$, 1070-1074 https://doi.org/10.1136/bjo.85.9.1070 (2001).

\section{Figures}

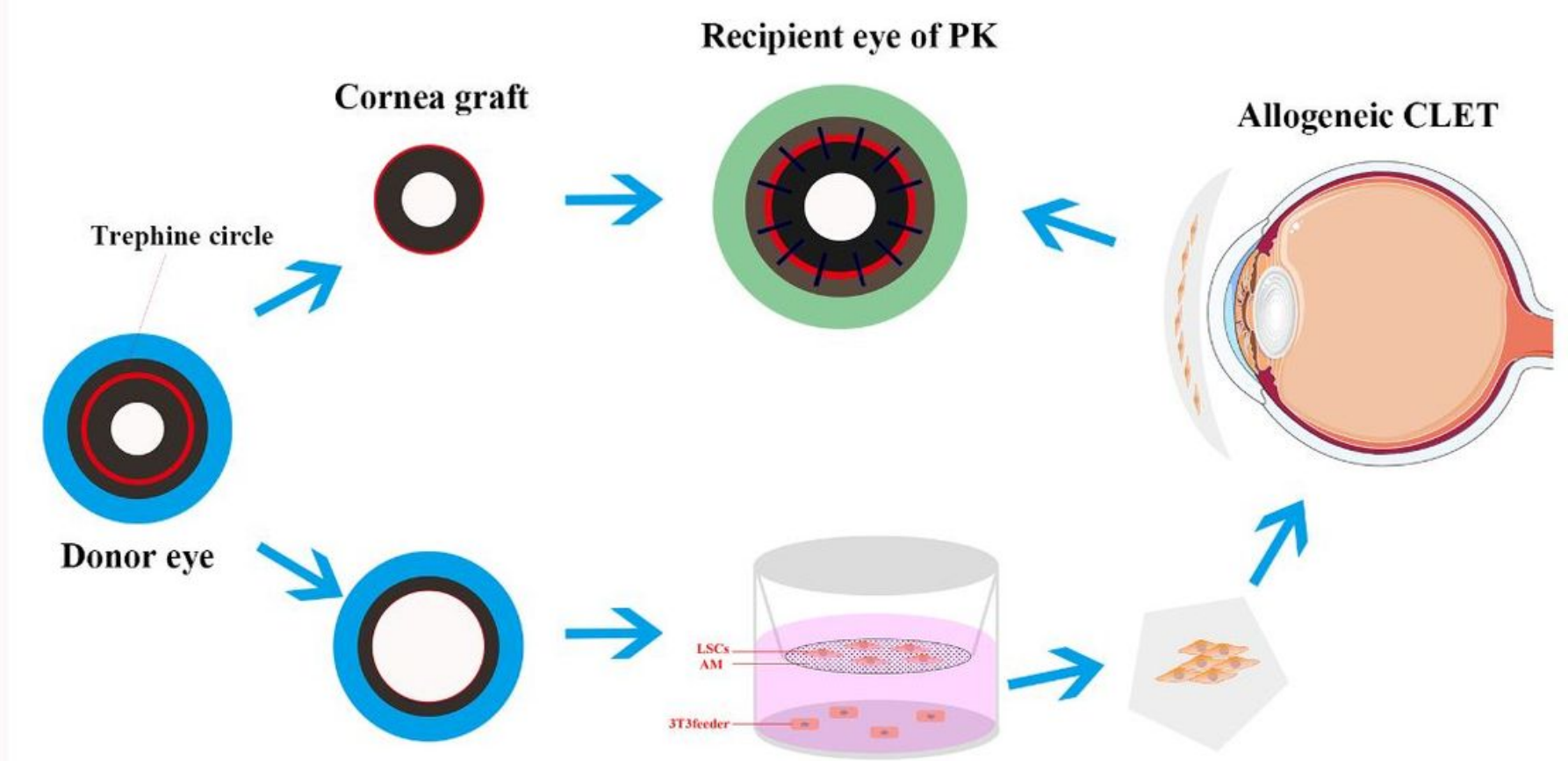

Donor corneoscleral ring Allogeneic stem cell culture Stem cell sheet

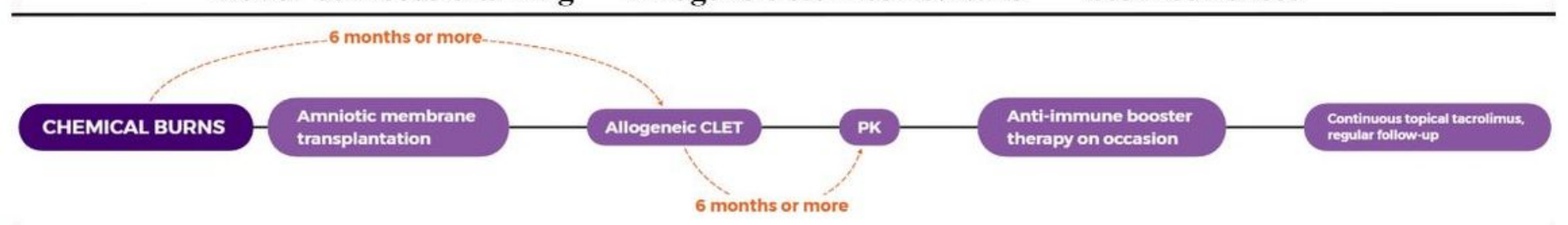

The procedure of penetrating keratoplasty following allogeneic CLET

Figure 1

The graphical procedures of PK following the allogeneic CLET strategy. The donated cornea tissue was trephined into a cornea graft using a 7.5-8.0 mm trephine and was used in PK surgery. The disposed corneoscleral ring after PK was cut into pieces, inoculated on a denuded AM laying on the bottom of transwell culture inserts, and then cocultured with MMC-inactivated NIH 3T3 fibroblasts. The stem cell sheet was then applied in the allogeneic CLET. After approximately 6 months, PK was performed in the same eye to improve central corneal transparency. A booster anti-rejection treatment (topical tacrolimus and fluorometholone, systemic hydrocortisone, prednisone, or cyclosporine if necessary) was given if needed after PK. Abbreviations: PK= penetrating keratoplasty, CLET= cultivated limbal epithelial transplantation, $\mathrm{AM}=$ amniotic membrane, $\mathrm{MMC}=$ mitomycin $\mathrm{C}$. Statement: this schematic diagram was originally illustrated by Junfa Xue (first author) using Adobe Photoshop CC (Adobe Systems Incorporated, USA). 
A

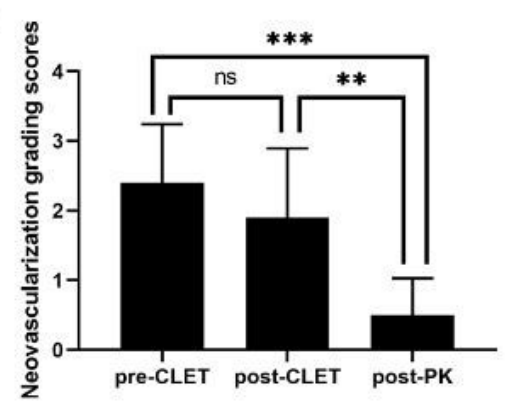

D

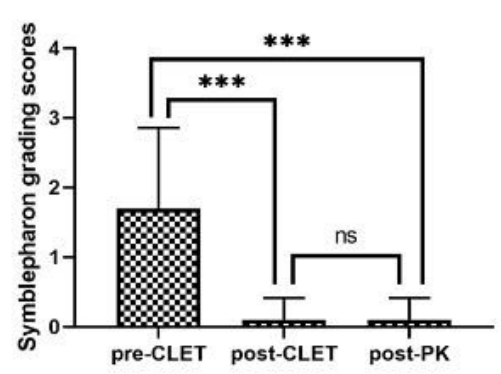

G

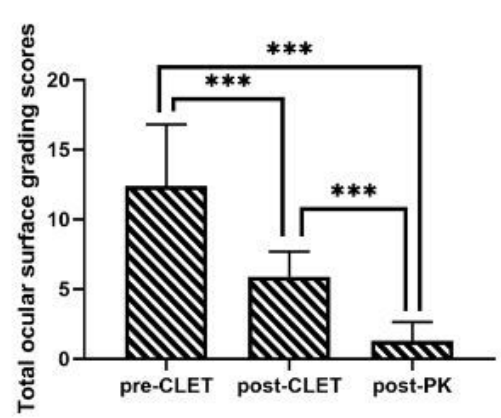

B

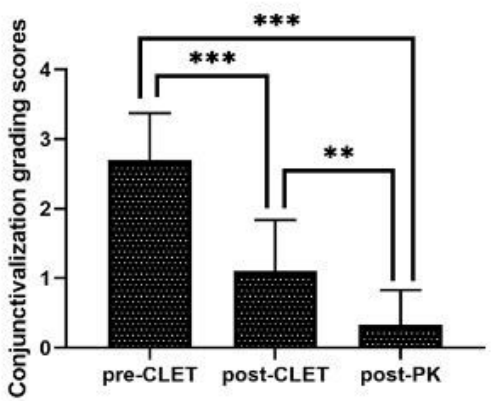

E

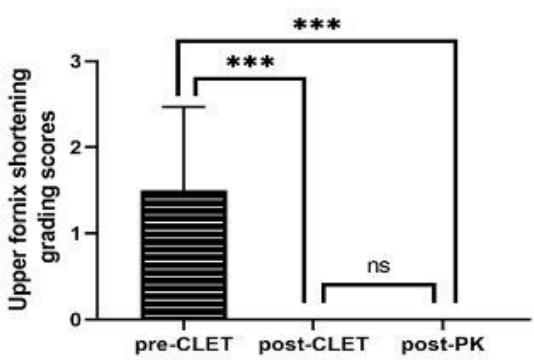

H

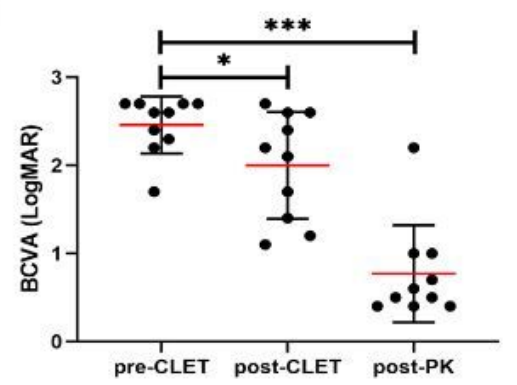

C

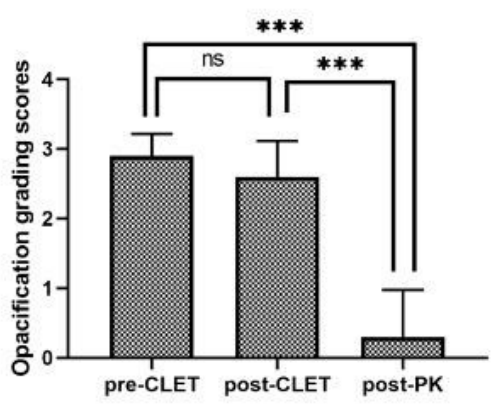

F

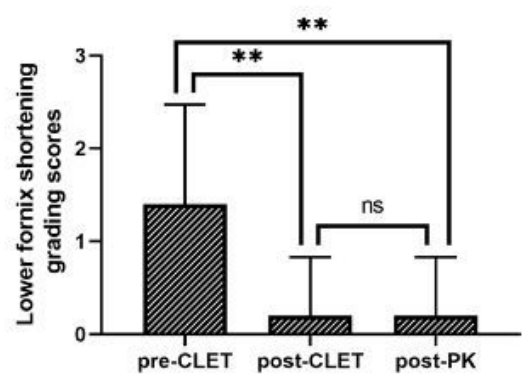

I

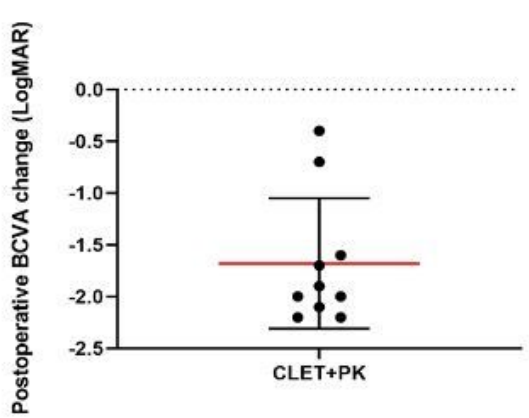

Figure 2

Outcomes of OSSs and the mean change in BCVA during the treatment course. (A): Corneal neovascularization grading scores (full scores: 3 ) were $2.40 \pm 0.84$ at baseline, decreased to $1.90 \pm 0.99$ after allogeneic CLET ( $P>0.05)$, and further decreased to $0.50 \pm 0.53$ after PK ( $P<0.01$, one-way ANOVA). (B): Corneal conjunctivalization grading scores (full scores: 3 ) were $2.70 \pm 0.67$ at baseline, declined to $1.10 \pm 0.74$ after allogeneic CLET $(P<0.001)$, and further decreased to $0.30 \pm 0.48$ after $P K(P<0.01$, one-way ANOVA). (C): Corneal opacification grading scores (full scores: 3 ) were $2.70 \pm 0.67$ at baseline, decreased to $2.60 \pm 0.52$ after allogeneic CLET $(P>0.05)$, and decreased to $0.30 \pm 0.67$ after PK $(P<0.001$, one-way ANOVA). (D): Symblepharon grading scores (full scores: 3$)$ were $1.70 \pm 1.16$ at baseline, decreased to $0.10 \pm 0.32$ after allogeneic CLET and were $0.1 \pm 0.32$ after $P K(X 2=18.664, P<0.001$, KruskalWallis test). (E): The upper fornix shortening grading scores (full scores: 3 ) were $1.50 \pm 0.97$ at baseline and then decreased to 0 after allogeneic CLET and PK $(X 2=24.027, P<0.001$, Kruskal-Wallis test). $(F)$ : The lower fornix shortening grading scores (full scores: 3$)$ were $1.40 \pm 1.07$ at baseline and then decreased to $0.20 \pm 0.63$ after allogeneic CLET and $P K(X 2=12.795, P<0.01$, Kruskal-Wallis test). (G): The total ocular surface grading scores (full scores: 18 ) were $12.40 \pm 4.40$ at baseline, declined to $5.90 \pm 1.80$ after allogeneic CLET, and then dropped to $1.40 \pm 1.51$ after $P K$ at the last follow-up $(X 2=20.406, P<0.001$, Kruskal-Wallis test). Black error bars represent standard deviations. $(\mathrm{H})$ : Comparison of BCVA at pre-CLET, post-CLET and post-PK $(2.46 \pm 0.32$, $1.83 \pm 0.65,0.77 \pm 0.55, X 2=19.356, P<0.001$, Kruskal-Wallis test). The red line shows the mean $B C V A$, and the black error bars represent the standard deviations. (I): The mean change in BCVA after CLET and PK. The red line shows the mean change in BCVA(-1.68 \pm 0.63$)$, and the black error bars represent $95 \%$ confidence intervals(Cl: $-2.00 \sim-1.27)$. Abbreviations: OSS= ocular surface grading scores, $\mathrm{CLET}=$ cultivated limbal epithelial transplantation, $\mathrm{PK}=$ penetrating keratoplasty. $\mathrm{BCVA}=$ best corrected visual acuity. ns $\mathrm{P}>0.05, * \mathrm{P}<0.05$, ** $\mathrm{P}<0.01$, *** $\mathrm{P}<0.001$. 

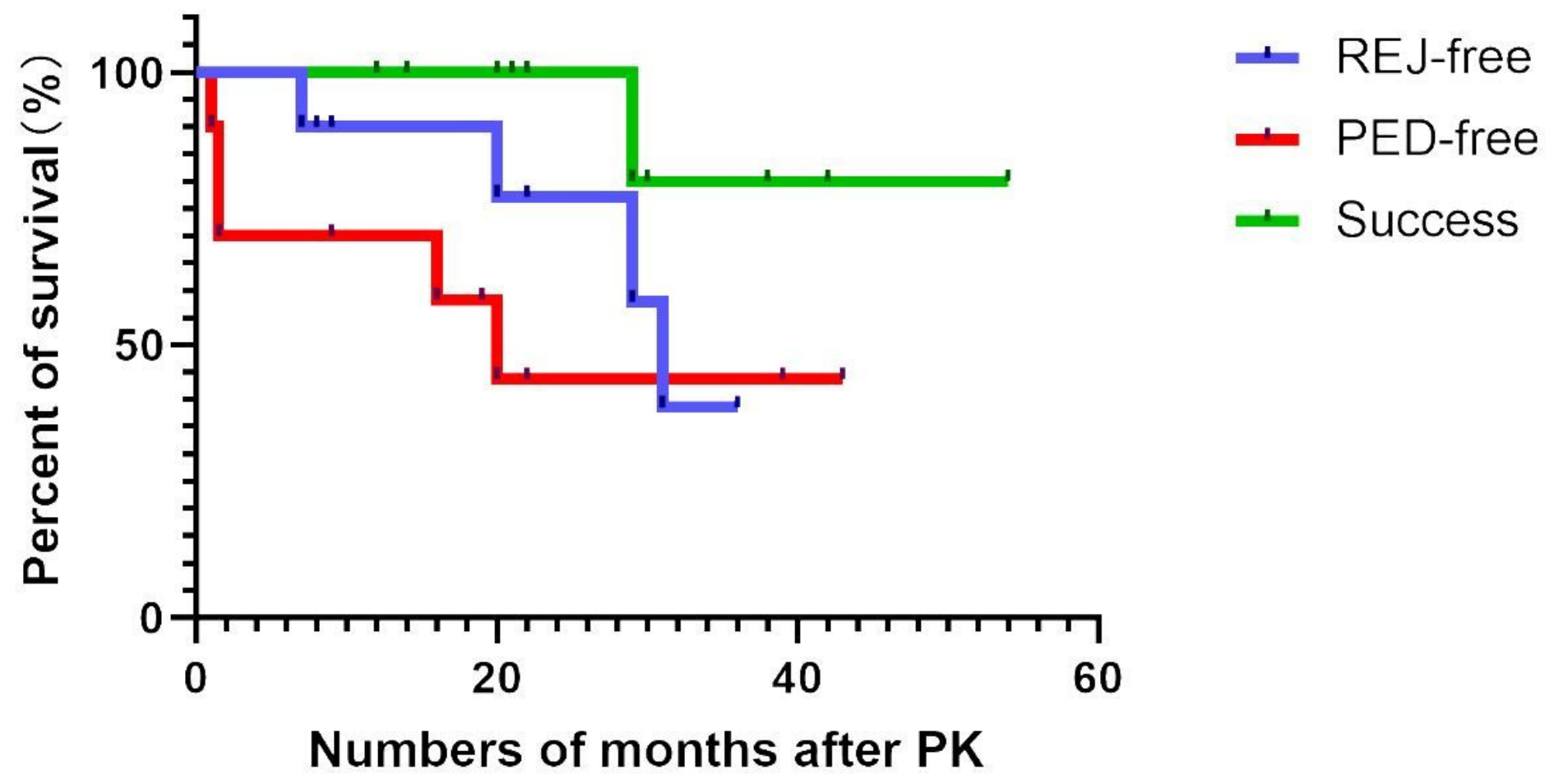

- Success

\section{Figure 3}

Kaplan-Meier survival analysis of rejection-free grafts, corneal PED-free grafts, and successful grafts. The PED events occurred at 1, 1.5, 1.5, 16, and 20 (mean $8.0 \pm 9.2$ ) months post-PK. Endothelial rejection events occurred at 7, 20, 29, and 36 (mean $23.0 \pm$ 12.5) months post-PK. The graft failure event occurred at 29 months after PK. Abbreviations: PED=persistent corneal epithelial defect, REJ= graft rejection, Success=transparent central graft survival, allogeneic CLET=allogenic cultivated limbal epithelial transplantation, $\mathrm{PK}=$ penetrating keratoplasty. 

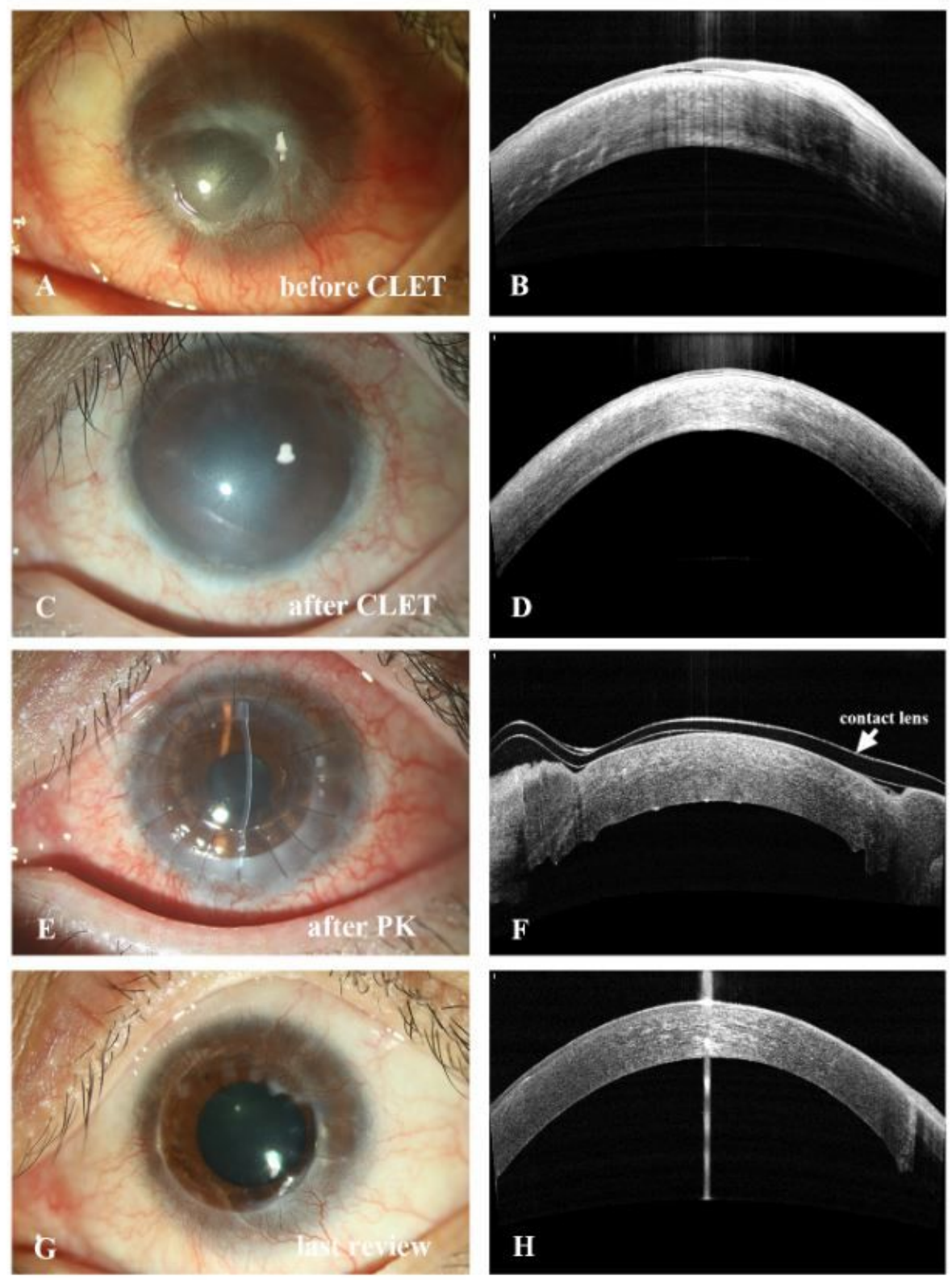

\section{Figure 4}

Case 2: A 27-year-old man injured by an alkali chemical burn in the left eye. (A, B) Six months after the injury, neovascularization (grade 3) with pannus reaching the central cornea and conjunctivalization (grade 3) were observed on the corneal surface. The BCVA decreased to 2.2 LogMAR. (C, D) A fully epithelized corneal surface with stromal neovascularization (grade 3) and fullthickness opacification (grade 2) can be detected three months after allogeneic CLET. The BCVA changed to 1.2 LogMAR. (E, F) Conjunctival congestion was still evident one month after PK. The corneal graft was clear with intact epithelium on the surface. The endothelial cell density was $2415 / \mathrm{mm} 2$. The BCVA was 0.4 LogMAR. (G, H) At last review, a stable ocular surface maintained. However, mild corneal neovascularization and opacification extended to the graft margin. The endothelial cell density was 990/mm2, and the BCVA was 0.5 LogMAR at the last visit. Abbreviations: BCVA=best-corrected visual acuity, CLET= cultivated limbal epithelial transplantation, PK=penetrating keratoplasty. 
HE
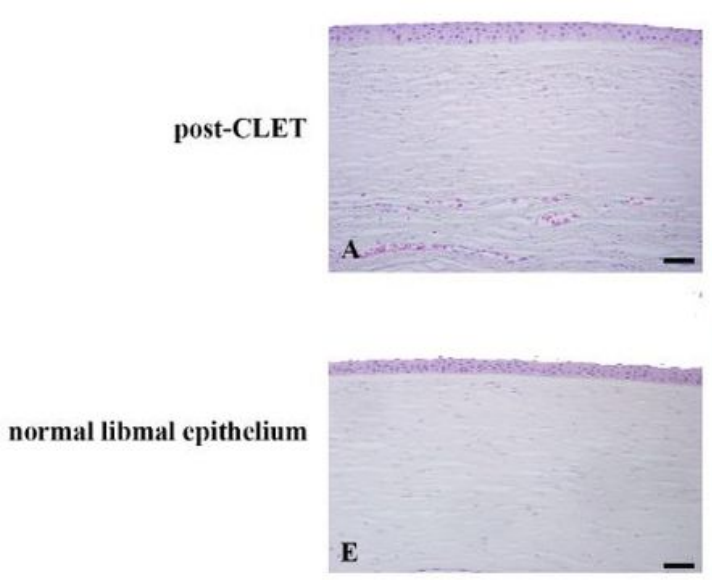

PAS
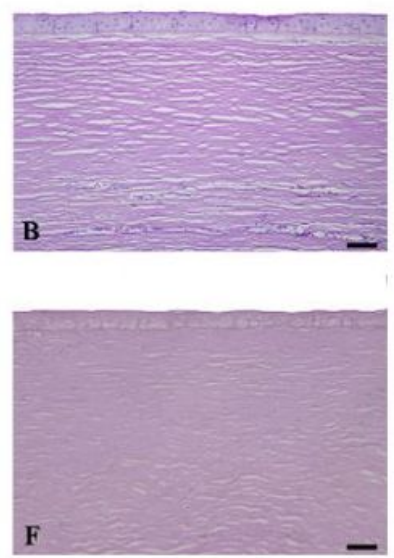

K3

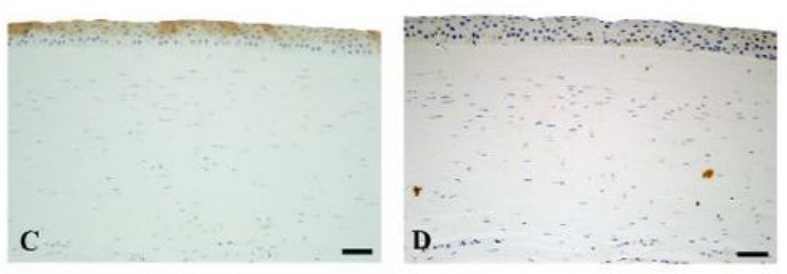

G

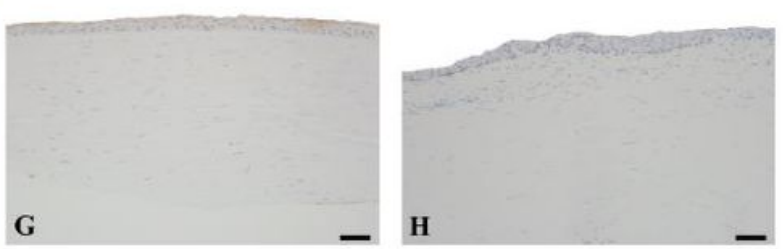

\section{Figure 5}

HE, Periodic acid-Schiff (PAS), and immunohistochemistry staining of corneal button samples after PK. Keratin 3 and P63 are visualized in brown. Magnification: 200x Abbreviations: CLET= cultivated limbal epithelial transplantation, PK=penetrating keratoplasty. Bar: 50um.

\section{Supplementary Files}

This is a list of supplementary files associated with this preprint. Click to download.

- supplementalmaterial.docx 